\title{
Spinal Epidural and Subarachnoid Abscess Due to Acute Pyelonephritis in a 56-Year-Old Woman: A Case Report and Literature Review
}

\author{
Da Ham Kim¹, Sung Hyun $\mathrm{Noh}^{2}$ \\ ${ }^{1}$ Department of Neurosurgery, National Health Insurance Service Ilsan Hospital, Goyang, Republic of Korea \\ ${ }^{2}$ Department of Neurosurgery, Ajou University Hospital, Ajou University College of Medicine, Suwon, Republic of Korea
}

\author{
Corresponding author: \\ Sung Hyun Noh \\ Department of Neurosurgery, Ajou \\ University Hospital, Ajou University \\ College of Medicine, 164, World \\ Cup-ro, Yeongtong-gu, Suwon \\ 16499, Republic of Korea \\ Tel: +82-31-219-4821 \\ Fax: +82-31-219-4822 \\ E-mail: juwuman11@nate.com
}

Received: June 2, 2021

Revised: July 16, 2021

Accepted: August 23, 2021
With recent advances in treatment technology, the mortality rate of spinal abscesses is decreasing. However, spinal abscesses are still unusual and difficult to treat. A 56-year-old woman presented with lower back pain and fever and chills that started two days before the visit. Initially, she showed lower limb extremity motor weakness. There was right focal acute pyelonephritis of the upper kidney portion with an associated complicated cyst on abdominal-pelvic computed tomography. Klebsiella pneumoniae was cultivated in blood and urine cultures. Spine magnetic resonance imaging with contrast enhancement was completed and the results showed epidural and subarachnoid space abscess formation in the whole lumbar space. The patient was treated with total laminectomy at L2-3 and laminotomy at L4-5 with epidural and subarachnoid abscess removal. After surgery, her back pain subsided enough that intravenous morphine was no longer needed. However, lower limb extremity motor weakness was not recovered. Antibiotics were administered to treat a potential cerebral nervous system infection for nine weeks. At the last follow-up, inflammation levels were normalized and the motor weakness in both legs was also normalized with active rehabilitation. This report described an epidural and subarachnoid abscess in a middle-aged woman due to urinary tract infection sepsis associated with acute pyelonephritis caused by Klebsiella pneumoniae. With proper surgery and adequate antibiotic treatment, the patient's symptoms improved significantly without any complications.

Key Words: Arachnoiditis; Epidural abscess; Pyelonephritis; Sepsis

\section{INTRODUCTION}

With recent advances in treatment technology, the mortality rate from spinal abscesses is decreasing. However, spinal abscesses are still unusual and difficult to treat ${ }^{3)}$. In general, spinal abscess occurs frequently in the epidural space, but rarely invade as intradural lesions ${ }^{2,7}$. According to the Massachusetts General Hospital between 1947 and 1974, the annual incidence of spinal epidural abscesses has risen in the past 2 to 3 decades from 0.2 to 1 case per 10,000 hospital admissions to 2.5 to 5.1 per 10,000 admissions ${ }^{7}$. This study reports the management of a 56-year-old woman with epidural and subarachnoid spinal abscesses due to acute pyelonephritis.

\section{CASE REPORT}

A 56-years-old woman with a medical history of acute hepatitis and osteoarthritis entered the hospital. She did not have diabetes mellitus, and there was no prior spinal intervention history. Her symptoms were lower back pain and pain in both flanks that started one day before the visit, and fever and chills that started two days before the visit. Initially, she showed lower limb extremity motor weakness as bilateral hip grade IIIN , bilateral knee grade IIIN, and bilateral big toe/ankle grade IV on the neurologic exam. On the admission date, she had a fever of $38^{\circ} \mathrm{C}$ and chills. On hospital day 1, costovertebral angle tenderness appeared in both flanks and the pain was more severe on the left than on the right side. An abdominal-pelvic computed tomography scan was performed. The results showed right focal acute pyelonephritis of the upper portion of the kidney. The blood inflammation markers were increased, showing an erythrocyte sedimentation rate (ESR) of $85 \mathrm{~mm} / \mathrm{hr}$ $(0-10 \mathrm{~mm} / \mathrm{hr})$, a C-reactive protein (CRP) level of $29.11 \mathrm{mg} / \mathrm{dL}$ (0-1 $\mathrm{mg} / \mathrm{dL}$ ). Klebsiella pneumoniae was cultivated in the blood and urine cultures. In the department of internal medicine, the antibiotic meropenem was administered.

Spine magnetic resonance imaging (MRI) was performed and the results showed an epidural abscess at the L2-3 level causing central canal compromise and a ventral dural tear at the $\mathrm{L} 3$ lower vertebral endplate level with an intradural extension of the abscess at the L3-S1 level and combined arachnoiditis (Fig. 1).

Surgical intervention was needed because of the lower limb ext- 

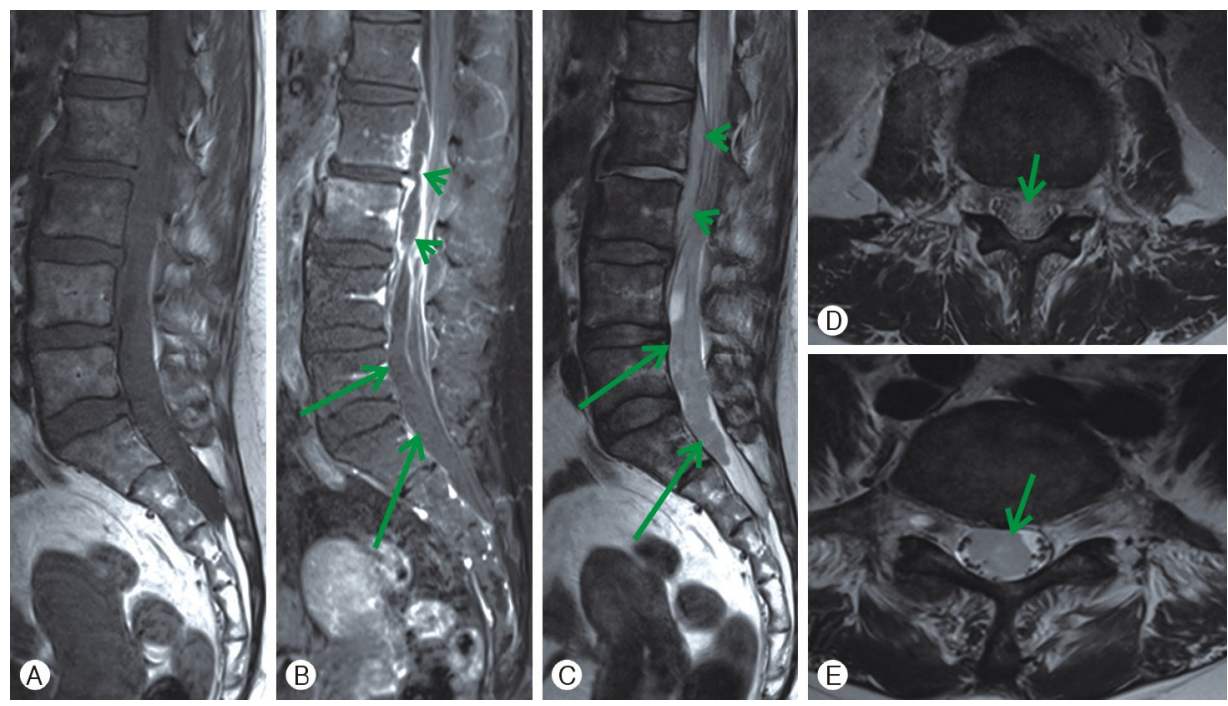

Fig. 1. (A) Preoperative magnetic resonance imaging (MRI) $\mathrm{Tl}$ image of the patient. (B, C) Preoperative lumbar-thoracic spine MRI T2 sagittal image and contrast-enhanced MRI T1 sagittal image show an epidural abscess at the L2-3 level causing central canal compromise and intradural extension of the abscess at the L3-S1 level and combined arachnoiditis. (D) MRI T2 axial image of the spine indicates a ventral dural tear at L3 lower vertebral endplate level. (E) MRI T2 axial image of the spine indicates the intradural extension of the epidural abscess to the L3-S1 level and combined arachnoiditis.
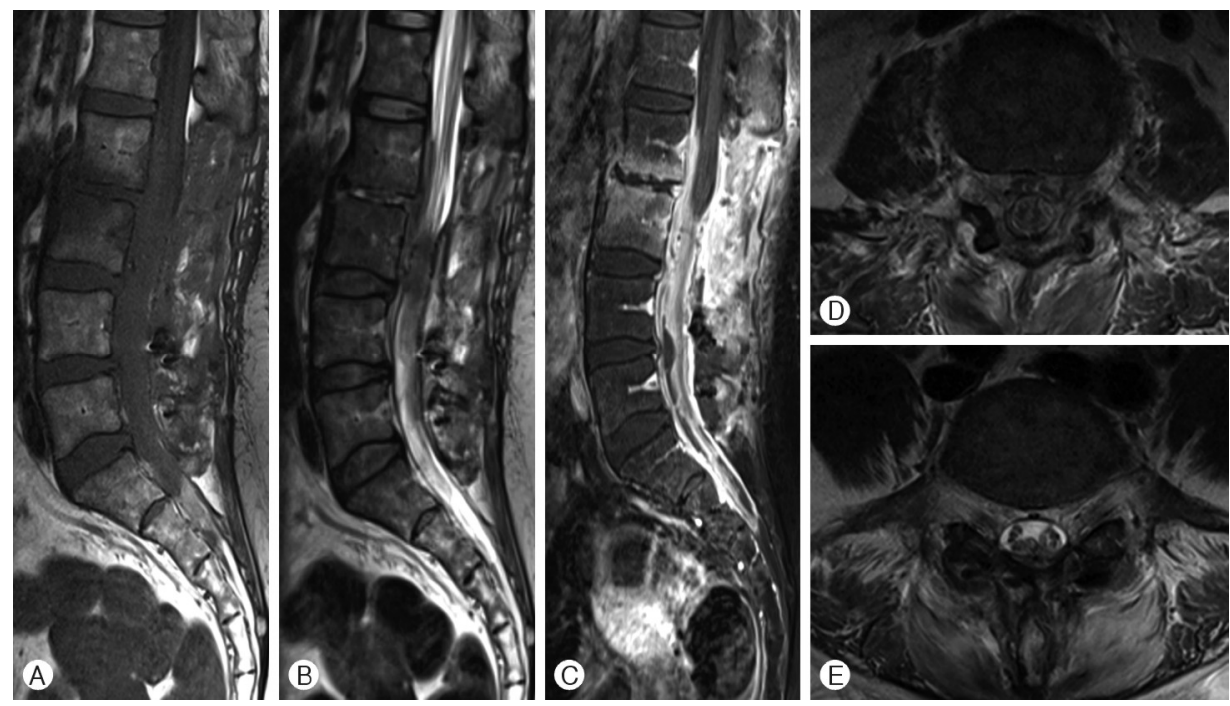

Fig. 2. (A) Postoperative magnetic resonance imaging (MRI) $\mathrm{Tl}$ image of the patient. $(\mathrm{B}, \mathrm{C})$ Postoperative lumbar-thoracic spine MRI T2 sagittal image and lumbar-thoracic spine contrast-enhanced MRI T1 sagittal image of the spine at follow-up 1 month after surgery show decreased prevertebral phlegmons and epidural abscesses at the L2-3 level. The postoperative changes also show remaining phlegmons in the epidural space and arachnoiditis at L4-5. (D) MRI T2 axial image of the spine indicates that the ventral dural tear at $\mathrm{L} 3$ lower vertebral endplate level is repaired and the epidural abscess is not seen. (E) MRI T2 axial image of the spine indicates that the decreased intradural extension of the epidural abscesses to the L3-S1 level.

remity motor weakness and the subdural empyema progression in spite of medical treatment. With the patient under general anesthesia, the surgery was performed with the patient in the prone position on the Jackson table. After confirming the surgical site on the radiograph, a dorsal midline incision was made. Total laminectomy at L2-3 and laminotomy at L4-5 were performed to removal the spinal 
abscess. At the L3 vertebral body lower margin level, a central ventral dura defect sized at $0.5 \times 0.5-\mathrm{cm}$ and cerebrospinal fluid leakage were confirmed. Bilateral L3 dorsal roołs swelling was seen. After irrigation, wrapping was performed around the dural defect using artificial dura. The surgery was completed uneventfully and the patient was taken to the Intensive Care Unit.

After surgery, back pain subsided enough. However, lower limb extremity motor weakness was not recovered. It was perhaps considered as the effect of dorsal roots swelling during removal of the spinal abscess. No growth was seen in the pus culture performed in the operation room, so antibiotics treatment was started as an unknown origin cerebral nervous system (CNS) infection. Ceftriaxone, vancomycin and metronidazole antibiotics were administered for the patient's CNS infection. No growth was seen in the follow-up blood culture. She exercised for seven weeks and was able to walk at the time of discharge. Three months after admission, a postoperative lumbar spine MRI with contrast enhancement was performed (Fig. 2). The MRI shows decreased prevertebral phlegmons and epidural abscesses at the L2-3 level with resolved central canal compromise and postoperative changes combined with remaining phlegmons in the epidural space and arachnoiditis at L4-5. At the last follow-up, the inflammatory markers decreased to ESR $21 \mathrm{~mm} / \mathrm{hr}$, and CRP $0.1 \mathrm{mg} / \mathrm{dL}$. From admission to discharge, meropenem, vancomycin, and metronidazole were administered for more than one week, and ceftriaxone was administered for nine weeks.

\section{DISCUSSION}

Spinal epidural abscesses are rare, but an epidural abscess accompanied by an intradural abscess is much rarer ${ }^{2,7}$. Mortality increases if the infection is not controlled by antibiotics or progresses further to become meningitis. Therefore, accurate diagnosis and treatment are very important. A spinal abscess is an infection, so using long-term antibiotics after abscess removal surgery is very important. Also, an extensive treatment course is needed to rehabilitate the patient.

Of the spinal abscess patients, $70 \%$ are men, and $68 \%$ of the women are 31 to 70 years old $\mathrm{d}^{7}$. The incidence in men was reported to be twice that in women ${ }^{7}$. The primary spinal abscess pathogen is Staphylococcus aureus in $70 \%$ of the cases and Streptococcus in $7 \%^{1,2,7}$. In our case, there was no growth in the abscess culture, but the blood and urine cultures indicated Klebsiella pneumoniae. This was a very unique case because it was different from the common reports of spinal abscesses.

Factors like diabetes mellitus, end-stage renal disease, septicemia, human immunodeficiency virus infection, malignancy, steroid use, intravenous drug abuse, alcoholism, distant site infection, and an indwelling catheter can cause a spinal abscess ${ }^{2,6,7}$. In our case, the patient had acute pyelonephritis, and the MRI showed osteomyelitis and prevertebral phlegmons on L2 and L3. The MRI showed a ventral dural tear at $\mathrm{L} 3$ of the lower vertebral endplate level, suggesting that the formation of the intradural abscess was through a dural tear.

Spinal abscesses are initially treated with antibiotics for a period of time. However, when neurological symptoms develop, decompressive surgical abscess removal is required ${ }^{5}$. Depending on the observed clinical and laboratory responses, such as ESR or CRP levels, some authors advocate only intravenous therapy for 6 to 8 weeks, while others suggest parenteral therapy for 6 to 8 weeks and oral therapy for more than 2 consecutive months ${ }^{4)}$. After successful surgery, we used antibiotics to target Klebsiella pneumoniae for 10 to 11 weeks and the patient was in a rehabilitation program. After rehabilitation, she was able to walk by herself. Although spinal abscesses are associated with high mortality, the patient was discharged affer a rehabilitation program, indicating a good outcome.

This report described an epidural and subarachnoid abscess in a middle-aged woman due to acute pyelonephritis caused by Klebsiella pneumoniae. With surgery and antibiotic treatment, the patient's symptoms improved significantly without any complications. And the motor weakness that occurred after surgery was greatly improved with active rehabilitation.

\section{CONFLICTS OF INTEREST}

No potential conflict of interest relevant to this article was reported.

\section{REFERENCES}

1. Chae HJ, Kim J, Kim C: Clinical characteristics of spinal epidural abscess accompanied by bacteremia. J Korean Neurosurg Soc 64:88-99, 2021

2. Cheon JE, Yang HJ, Chung YN, Park SB: Pyogenic intradural abscess of lumbar spine: A case report. Korean J Neurotrauma 11:18-21, 2015

3. Lim HY, Choi HJ, Kim S, Kuh SU: Chronic spinal subdural abscess mimicking an intradural-extramedullary tumor. Eur Spine J 22 Suppl 3:S497-S500, 2013

4. Luzzati R, Giacomazzi D, Danzi MC, Tacconi L, Concia E, Vento S: Diagnosis, management and outcome of clinicallysuspected spinal infection. J Infect 58:259-265, 2009

5. Ma H, Kim I: Clinical outcomes of spinal epidural abscess. Korean J Spine 9:6-11, 2012

6. Sharfman ZT, Gelfand Y, Shah P, Holtzman AJ, Mendelis JR, Kinon MD, et al.: Spinal epidural abscess: A review of presentation, management, and medicolegal implications. Asian Spine J 14:742-759, 2020

7. Tompkins M, Panuncialman I, Lucas P, Palumbo M: Spinal epidural abscess. J Emerg Med 39:384-390, 2010 